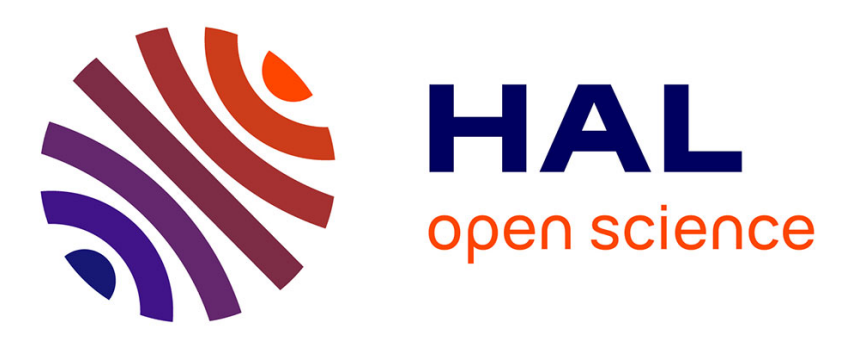

\title{
Umpolung Reactivity of in Situ Generated Phosphido-Boranes: An Entry to P-Stereogenic Aminophosphine-Boranes
}

Sébastien Lemouzy, Romain Membrat, Enzo Olivieri, Marion Jean, Muriel Albalat, Didier Nuel, Laurent Giordano, Damien Hérault, Gérard Buono

\section{To cite this version:}

Sébastien Lemouzy, Romain Membrat, Enzo Olivieri, Marion Jean, Muriel Albalat, et al.. Umpolung Reactivity of in Situ Generated Phosphido-Boranes: An Entry to P-Stereogenic AminophosphineBoranes. Journal of Organic Chemistry, 2019, 84, pp.4551-4557. 10.1021/acs.joc.9b00333 . hal02079962

\section{HAL Id: hal-02079962 https://hal.science/hal-02079962}

Submitted on 23 Mar 2020

HAL is a multi-disciplinary open access archive for the deposit and dissemination of scientific research documents, whether they are published or not. The documents may come from teaching and research institutions in France or abroad, or from public or private research centers.
L'archive ouverte pluridisciplinaire HAL, est destinée au dépôt et à la diffusion de documents scientifiques de niveau recherche, publiés ou non, émanant des établissements d'enseignement et de recherche français ou étrangers, des laboratoires publics ou privés. 


\section{Umpolung Reactivity of in Situ Generated Phosphido-Boranes: An Entry to P-Stereogenic Aminophosphine-Boranes}

Lemouzy, S., Membrat, R., Olivieri, E., Jean, M., Albalat, M., Nuel, D., Giordano, L., Herault, D., Buono, G.

ABSTRACT: The synthesis of P-stereogenic aminophosphine-boranes has been developed on the basis of umpolung reactivity of in situ generated alkylarylphosphido-boranes, which are normally configurationally unstable intermediates. In our case, their high configurational stability was due to the slow release of the hydroxyalkyl protecting group, together with the fast formation of the iodophosphanylborane in the presence of $\mathrm{N}$-iodosuccinimide. The subsequent substitution reaction was found to proceed in moderate to good yields and in a very high stereospecifity (es) using a variety of amines as nucleophiles.

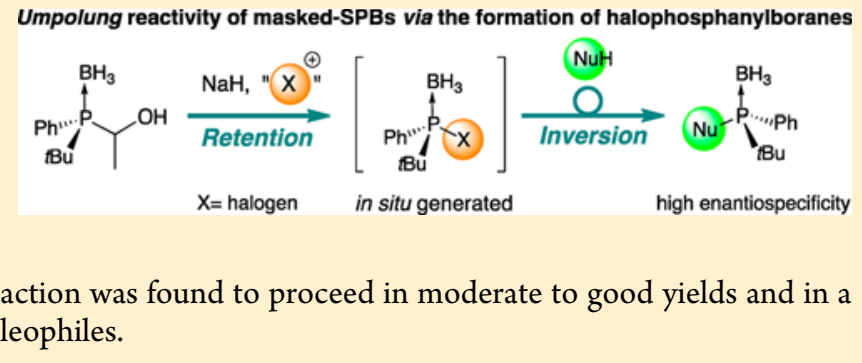

Umpolung reactivity of masked-SPBs via the formation of halophosphanylboranes he access to P-stereogenic compounds is crucial for the development of catalytic enantioselective methodologies, as these molecules are useful both in asymmetric transitionmetal-mediated catalysis and organocatalysis. ${ }^{1}$ Moreover, Pstereogenic phosphines have recently known a growing interest, allowing the development of enantioselective transformations using cheap and environmentally benign transitionmetal catalysts. ${ }^{2}$ Although P(III) compounds and more specifically phosphines have received particular attention, ${ }^{3}$ the use of $\mathrm{P}(\mathrm{V})$ preligands for transition-metal catalysis has emerged as a convenient alternative to air-sensitive trialkylphosphines (Scheme 1).

Thus, secondary phosphine oxides ${ }^{4}$ and iminophosphoranes ${ }^{5}$ have appeared to be interesting preligands, owing to their stability and reactivity of their metal complexes, by taking advantage of the $\mathrm{XH} / \mathrm{PH}$ tautomerism of the $\mathrm{P}(\mathrm{V})$

Scheme 1. $\mathrm{P}(\mathrm{V})$ Preligands: An Alternative to $\mathrm{P}(\mathrm{III})$ Ligands

Phosphine ligands:

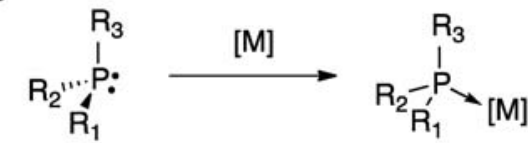

Unstable to air if

$R_{1}, R_{2}$ and $R_{3}=A l k y l$

SPO and iminophosphorane preligands:

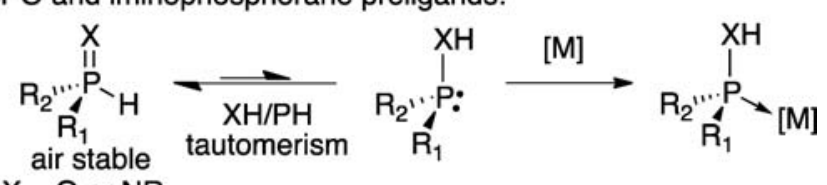

compounds. In recent years, P-stereogenic iminophosphorane preligands applied in asymmetric catalysis displayed excellent selectivities in enantioselective Pauson-Khand, ${ }^{6}$ cycloisomerization, $^{5 \mathrm{~b}}$ or hydrogenation ${ }^{5 \mathrm{~g}, 7}$ reactions. Despite their efficiency in asymmetric catalysis, these compounds have been overlooked by the scientific community for a long time. This is mostly due to the scarce available methods to synthesize P-stereogenic compounds, ${ }^{8,9}$ including P-stereogenic nitrogen-substituted compounds. An efficient access to P-stereogenic molecules relies on the use of electrophilic phosphorus-borane compounds through their umpolung reactivity. The modular synthesis of P-stereogenic compounds using chlorophosphanylborane intermediates, generated by acidolysis of aminophosphine-boranes (APB) through inversion of configuration at the phosphorus center, was first reported by Jugé and co-workers ${ }^{10}$ (Scheme 2). Thus, a large variety of aryl(alkyl) and diaryl P-stereogenic molecules can be accessed with carbon, nitrogen, oxygen, and sulfur nucleophiles. Imamoto and co-workers also reported such reactivity of secondary phosphido-boranes to generate electrophilic molecules and showed that the stereochemistry of the nucleophilic substitution with organometallic reagents is dependent on the hybridization of the carbon atom. ${ }^{11}$ However, the isolation and handling of halophosphanylboranes remains erratic, ${ }^{10 a}$ so these have been replaced with more convenient phosphorus reagents. Indeed, our group first described the use of $O$-mesylated phosphinite-boranes (easily available from phosphinous acid-boranes), as electrophilic precursors to access enantioenriched secondary phosphineboranes (SPBs). ${ }^{12}$ Afterward, the group of Verdaguer has depicted the first general stereospecific synthesis of APB using a similar method. ${ }^{3 a, 13}$ The authors showed the high efficiency

configurationally stable species 
Scheme 2. Previous Examples of Electrophilic or Nucleophilic Reactivity of Phosphorus-Boranes

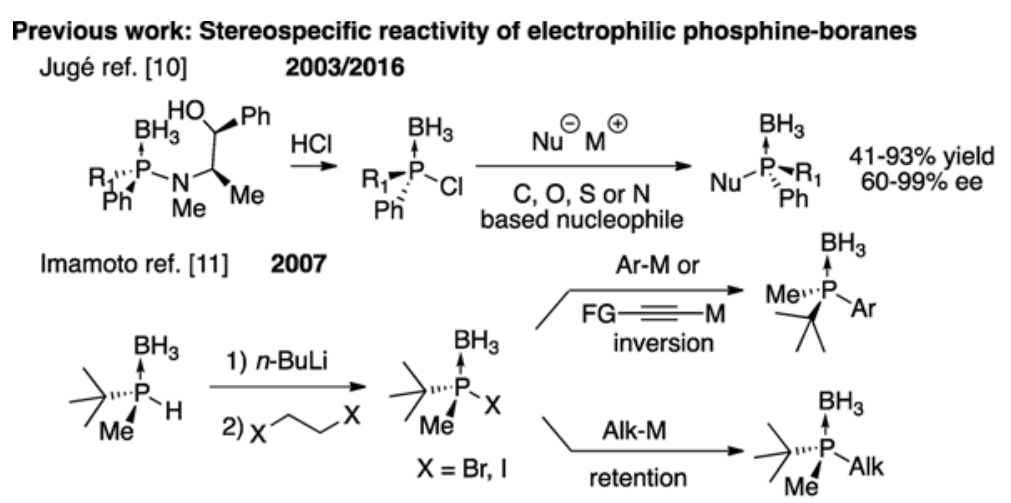

Our group ref. [12] $2011 /$ Verdaguer ref. [13] 2015

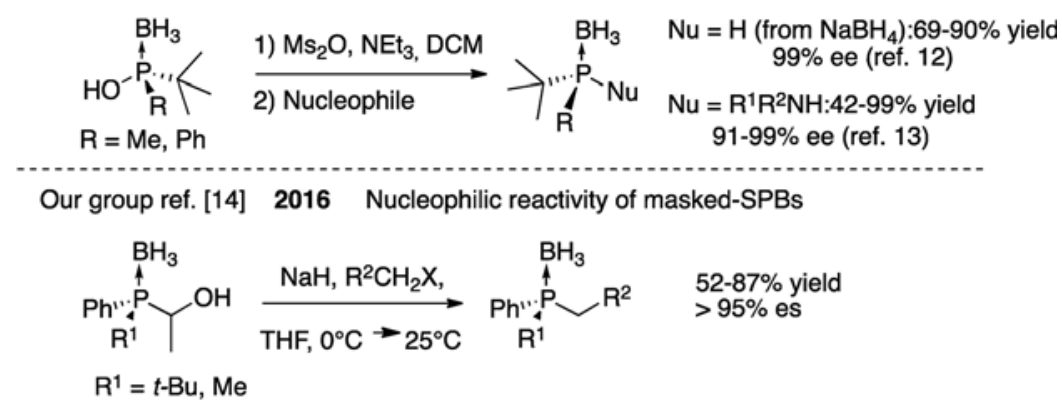

This work: Umpolung reactivity of masked-SPBs through halophosphanylboranes

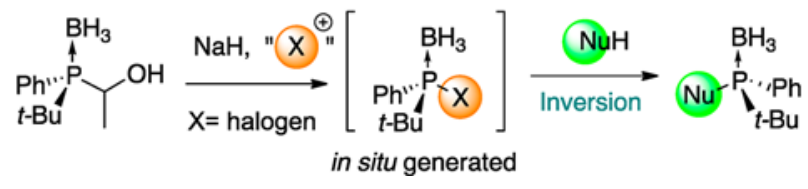

of such MaxPHOX ligands in asymmetric iridium-catalyzed hydrogenation of alkenes and imines. ${ }^{5 \mathrm{~g}, 7 \mathrm{~b}}$ Lately, we described a protecting-group-based approach for the alkylation of masked SPBs, through the in situ generation of configurationally stable and reactive alkyl(aryl) phosphido-boranes species. ${ }^{14}$ However, the use of the latter limits the extension of this methodology to other P-stereogenic compounds, as only carbon electrophiles were found to be effective for this reaction.

In order to increase the diversity in substitution pattern within the alkylarylphosphorus-borane products, we envisioned an umpolung process, which could ideally occur under basic conditions with a compatible stoichiometric oxidant and a nucleophilic reagent. To test our hypothesis, we conducted the reaction of substrate $\left(R_{\mathrm{P}}\right)-\mathbf{1}$, in the presence of $\mathrm{NaH}, \mathrm{N}$ iodosuccinimide (NIS), and benzylamine, which afforded the expected compound 2a (Tables 1 and 2). Thus, we first evaluated the influence of the solvent on both reactivity and stereospecificity of the transformation (Table 1). Ether solvents $\left(\mathrm{Et}_{2} \mathrm{O}\right.$ and $\mathrm{THF}$, entries $\left.1-3\right)$ allowed the selective formation of $\left(S_{\mathrm{P}}\right)-\mathbf{2 a}$, and THF proved superior to any erosion of the chiral information at the phosphorus atom. In order to rationalize these observations, we hypothesized that the separation of the sodium phosphido-borane ion pair was responsible for this loss of chiral information at the phosphorus atom. ${ }^{15}$ Although the full conversion of $\left(R_{\mathrm{P}}\right)-\mathbf{1}$ was observed in all cases, the presence of numerous degradation byproducts (resulting mostly from the deboronation/phosphorus oxida-
Table 1. Solvent Screening

\begin{tabular}{|c|c|c|c|c|c|}
\hline $\begin{array}{r}P h " \\
\text { Phi } \\
t-B i \\
( \\
\text { e.r. }\end{array}$ & $\mathrm{OH}$ & $\begin{array}{l}\text { 1) } \mathrm{NaH}_{2} \\
\text { 2) } \mathrm{NIS} 1 \\
\text { 3) } \mathrm{BnNH}_{2} \\
\text { Solvent, }\end{array}$ & $\begin{array}{l}\text { uiv. } \\
\text { div. } \\
\underset{\text { equiv. }}{\longrightarrow}-25^{\circ} \mathrm{C}\end{array}$ & $\left(S_{p}\right)$ & $\begin{array}{l}3 \mathrm{H}_{3} \\
\ldots \mathrm{Ph} \\
t-\mathrm{Bu}\end{array}$ \\
\hline entry & solvent & $t(\mathrm{~h})$ & yield $(\%)^{b}$ & er & es $(\%)^{c}$ \\
\hline 1 & $\mathrm{Et}_{2} \mathrm{O}$ & 16 & 47 & $88: 12$ & 84 \\
\hline 2 & THF & 16 & 43 & $94: 6$ & 98 \\
\hline 3 & THF & 1 & 71 & $94: 6$ & 98 \\
\hline 4 & DMF & 16 & 48 & $50: 50$ & 0 \\
\hline 5 & DMAc & 16 & 23 & $50: 50$ & 0 \\
\hline
\end{tabular}

${ }^{a}$ Compound $1: \mathrm{dr} \approx 1: 1$, er $=95: 5$ (both diastereomers). ${ }^{b}$ After column chromatography. In all cases, full conversion of 1 was observed. ${ }^{c}$ Stereospecificity: es $\%=$ ee $(\mathbf{2 a}) /$ ee $(\mathbf{1})$; ee determined by chiral HPLC.

tion of $\mathbf{1}$ and $\mathbf{2 a}$ ) dropped down the proportion of $\mathbf{2 a}$ to $60 \%$ at best. The use of highly polar solvents favoring the ion pair dissociation (DMF and DMAc, entries 4 and 5) resulted in the full racemization of the desired product.

We were pleased to find that the complete conversion of 1a was observed after a $1 \mathrm{~h}$ reaction, and consequently, the formation of byproducts dramatically decreased (entry 3 ). Under these conditions, $71 \%$ of $\mathbf{2 a}$ could be isolated, with a 
Table 2. Base and Oxidant Screening

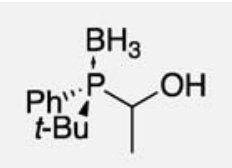

$\left(R_{\mathrm{P}}\right)-1$

e.r. $=95: 5^{a}$
1) Base 2 equiv.

2) $[\mathrm{Ox}] 1$ equiv.

3) $\mathrm{BnNH}_{2} 1$ equiv. THF, $0 \rightarrow 25^{\circ} \mathrm{C}$ $1 \mathrm{~h}$

\begin{tabular}{lclc} 
[ox] & yield $(\%)^{b}$ & \multicolumn{1}{c}{ er } & es $(\%)^{c}$ \\
NIS & 71 & $94: 6$ & 98 \\
NIS & 77 & $83: 17$ & 73 \\
NIS & $d$ & nd & nd \\
NIS & $d$ & nd & nd \\
NBS & 69 & $88: 12$ & 95 \\
NCS & 17 & $90.5: 9.5$ & 90 \\
I $_{2}$ & 68 & $77: 23$ & 60
\end{tabular}

${ }^{a}$ Compound $1: \mathrm{dr} \approx 1: 1$, er $=95: 5$ (both diastereomers). ${ }^{b}$ After column chromatography. In all cases, full conversion of 1 was observed. ${ }^{c}$ Stereospecificity: es $\%=$ ee $(\mathbf{2 a}) /$ ee $(\mathbf{1})$; ee determined by chiral HPLC. ${ }^{d}$ No presence of $\mathbf{2 a}$ was detected on the ${ }^{31} \mathrm{P}$ NMR of the crude. ${ }^{e}$ Compound $1: \mathrm{dr} \approx 1: 1$; er $=90: 10$ (both diastereomers) was used as the substrate.

complete stereospecificity ( $\mathrm{er}=94: 6$, es $=98 \%$ ). The absolute configuration of $\left(S_{\mathrm{p}}\right)$-2a was established by X-ray diffraction. (See the Supporting Information, Figure S1, pages S50-S51, for details.) Overall, the reaction proceeds with inversion of configuration, as result of retention of configuration within the halogenation step and the subsequent inversion of the substitution reaction with the amine.

In order to increase the yield of $2 \mathbf{a}$, we then turned our attention on the screening of the nature of both oxidant and base (Table 2). Although $\mathrm{Me}_{3} \mathrm{SiONa}$ proved to be slightly better compared to $\mathrm{NaH}, \mathbf{2 a}$ was isolated with a substantial racemization in this case (entry 2). On the other hand, the use of weaker sodium-based inorganic bases (entries 3 and 4) did not lead to the formation of the desired product. Having established $\mathrm{NaH}$ as the base of choice, we examined compatible halogenated oxidants. N-Bromosuccinimide (NBS) proved to be similar compared to NIS in terms of efficiency and stereospecificity (entries 1 and 5), while $\mathrm{N}$ chlorosuccinimide (NCS) was less efficient, favoring undesired byproducts under the same reaction conditions. In this case, the increased oxidation potential of NCS (compared with NBS and NIS) may account for this result.

Finally, molecular iodine showed a similar reactivity (entry 7) compared to NIS and NBS; however, substantial racemization of the desired product was observed. This racemization can be rationalized with the increase of iodide anion concentration in the reaction media, which may kinetically favor the racemization by nucleophilic attack of an iodide anion on the iodophosphanylborane intermediate. ${ }^{16}$ This result further supports the hypothesis of an iodophosphanylborane intermediate (together with the observed inversion of configuration at the phosphorus center). Using the best conditions (Table 2, entry 1 ), 2 a could be obtained in a good yield $(71 \%)$ without erosion of the chiral information on the phosphorus atom. With these conditions in hand, we evaluated the scope of this transformation, by reacting the substrate $\left(S_{\mathrm{P}}\right)$-1 with a variety of amine nucleophiles. All reactions proceeded cleanly, and various benzylamines, pentylamine, and even ammonia were well tolerated (Figure 1).

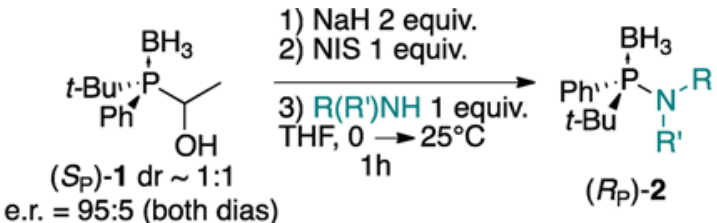

Reactive nucleophiles:

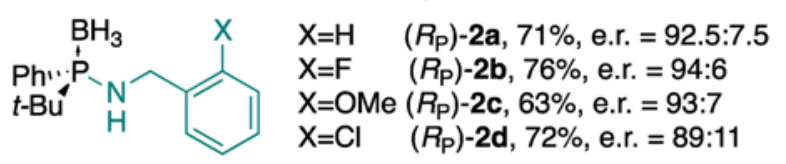<smiles>[B][PH]([18OH])([Pb])NCc1cccc(OC)c1</smiles>

$\left(R_{\mathrm{P}}\right)-2 \mathrm{e}, 76 \%$, e.r. $=87.5: 12.5$<smiles>CCCCCN[PH]([13CH2])([13CH3])[135I]</smiles>

$\left(R_{\mathrm{P}}\right)-\mathbf{2 g}, 43 \%$, e.r. $=88.5: 11.5$

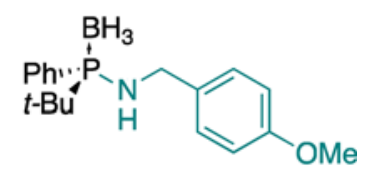

$\left(R_{\mathrm{P}}\right)-\mathbf{2 f}, \mathbf{8 1 \%}$, e.r. $=95: 5$<smiles>N[Pb]([18OH])[Pb]</smiles>

$\left(R_{\mathrm{P}}\right)-\mathbf{2 h}, 40 \%$, e.r. $=81: 19$

\section{Unreactive nucleophiles:}

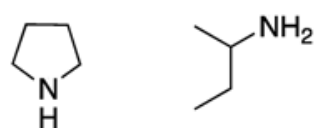

Figure 1. Scope of the umpolung reactivity of masked phosphidoboranes.

Compound $\left(R_{\mathrm{P}}\right)$-2a was obtained with a similar er as $\left(S_{\mathrm{P}}\right)$-2a (Table 1 , entry 3$)$, revealing the stereospecificity of the reaction. The best result ( $81 \%$ yield) was obtained with $\mathbf{2 f}$ since no racemization was observed. The yields are slightly affected by the electronic and steric effects of substituents. Pentylamine featured a lower reactivity than benzylamines to afford $2 \mathrm{~g}$ with a $43 \%$ yield. The reaction of $\left(S_{\mathrm{p}}\right)-1$ with ammonia ( $0.4 \mathrm{M}$ in $\mathrm{THF}$ ) gave $\mathbf{2 h}$ with a moderate yield $(40 \%)$ and with a slightly decreased enantioselectivity (er = 81:19), when compared to those obtained by Verdaguer. ${ }^{5 \mathrm{a}}$ The formation of product $(+)-\mathbf{2 h}$ confirms the absolute configuration $\left(R_{\mathrm{P}}\right)$ of our product. Hindered primary amines or secondary amines were found to be unreactive nucleophiles under these conditions in agreement with the stereochemical features of the bulky intermediate.

In conclusion, we have shown that the recent strategy developed for the $P$-alkylation of stable phosphido-boranes can be applied to the synthesis of enantioenriched APB. Indeed, in situ removal of a hydroxyalkyl moiety under mild basic conditions enabled the generation of these highly reactive and normally unstable phosphorus species. The further halogenation step in the reaction media allowed us to circumvent the low configurational stability of these transient species and to generate in situ these "umpoled" reactive halophosphanylboranes. Their rapid reaction with amines allowed the efficient and versatile synthesis of a variety of APB. The application of these preligands in enantioselective transformations, as well as the extension of this approach to other nucleophiles, is currently underway in our laboratory. 


\section{EXPERIMENTAL SECTION}

General Information. All solvents were purified by standard procedures or obtained from a solvent purification system (Braun SPS 800). Unless otherwise mentioned, all reactions were carried out under an atmosphere of dry argon. Thin-layer chromatography (TLC) was carried out on Merck silica gel $60 \mathrm{~F}_{254}$ and visualized under ultraviolet light (254 and $366 \mathrm{~nm}$ ), or through spraying with $5 \%$ phosphomolybdic acid in $\mathrm{EtOH}, \mathrm{H}_{2} \mathrm{SO}_{4}$-acidified $p$-anisaldehyde solution in EtOH or by placing in iodine vapor. Flash chromatography was performed with Merck silica gel 60 (230-400 mesh). Solvents for chiral chromatography ( $n$-hexane, $n$-heptane, 2-PrOH, EtOH, $\mathrm{MeOH}$ ) were HPLC grade, degassed, and filtered on Millipore membrane $0.45 \mu \mathrm{m}$ before use. Lux-Cellulose-4, Lux-Cellulose-3, LuxCellulose-2, $(S, S)$-Whelk-O1, Chiralcel OD-3 and Chiralpak IG, AS$\mathrm{H}$, IB columns $(250 \mathrm{~mm} \times 4.6 \mathrm{~mm})$ were used for the analytical separation. Chiral HPLC analyses were performed on a screening unit composed of a Merck D-7000 system manager, Merck-Lachrom L7100 pump, Merck-Lachrom L-7360 oven, Merck-Lachrom L-7400 UV detector, and Jasco OR-1590 polarimetric or Jasco CD-1595 circular dichroism detector. Semipreparative HPLC separations were performed with a Merck-Hitachi LiChrograph L-6000 pump, MerckHitachi L-4000 UV detector, and Merck D-7000 system manager. Retention times $\left(t_{\mathrm{R}}\right)$ are given in minutes, retention factor $k_{\mathrm{i}}=\left(t_{\mathrm{R}_{\mathrm{i}}}-\right.$ $\left.t_{\mathrm{R}_{0}}\right) / t_{\mathrm{R}_{0}}$, and enantioselectivity factor $\alpha=k_{2} / k_{1}$. The sign given by the chiroptical detector is the sign of the enantiomer in the mobile phase used, at the specified wavelength. ${ }^{1} \mathrm{H},{ }^{13} \mathrm{C}$, and ${ }^{31} \mathrm{P}$ NMR spectra were recorded on Bruker Avance III nanobay spectrometers operating at 400 and $300 \mathrm{MHz}$ for ${ }^{1} \mathrm{H} .{ }^{13} \mathrm{C}$ and ${ }^{31} \mathrm{P}$ nuclei were observed with ${ }^{1} \mathrm{H}$ decoupling. Unless otherwise specified, NMR spectra were performed in $\mathrm{CDCl}_{3}$. As an external reference for ${ }^{31} \mathrm{P}$ NMR spectra, $85 \%$ phosphoric acid was used. Chemical shifts $(\delta)$ of ${ }^{1} \mathrm{H}$ and ${ }^{13} \mathrm{C}$ are reported in ppm relative to $\mathrm{CHCl}_{3}\left(\delta=7.26\right.$ for ${ }^{1} \mathrm{H}$ and $\delta=77.0$ for $\left.{ }^{13} \mathrm{C}\right)$ and $\mathrm{C}_{6} \mathrm{D}_{6}\left(\delta=7.15\right.$ for ${ }^{1} \mathrm{H}$ and $\delta=128.02$ for $\left.{ }^{13} \mathrm{C}\right)$. Coupling constants $(J)$ are given in hertz $(\mathrm{Hz})$. Proton $\left({ }^{1} \mathrm{H}\right)$ NMR information is given in the following format: multiplicity ( $(s$, singlet; $d$, doublet; $t$, triplet; q, quartet; sept; septet; $\mathrm{m}$, multiplet), coupling constant $J$, number of protons). The prefix broad or $\mathrm{b}$ indicates the signal in question is broadened. Melting points (uncorrected) were determined in a capillary tube with a Mettler LP61 apparatus. $[\alpha]_{\mathrm{D}}^{25}$ values were determined with a PerkinElmer Polarimetric 341. High-resolution MS experiments were performed with a QStar Elite mass spectrometer (Applied Biosystems SCIEX, Concord, ON, Canada) equipped with an electrospray ionization (ESI) source. In the positive ion mode, the capillary voltage was set at $+5500 \mathrm{~V}$ and the cone voltage was set between 10 and $55 \mathrm{~V}$. In this hybrid instrument, ions were measured using an orthogonal acceleration time-of-flight (oa-TOF) mass analyzer. In MS, accurate mass measurements were performed using two reference ions from a poly(ethylene glycol) or poly(propylene glycol) internal standard, according to a procedure described elsewhere.

Synthesis of Starting Material 1. All of the starting phosphineboranes materials were made according to our previously reported procedure. ${ }^{17}$

$\left(S_{\mathrm{P}}\right)$-tert-Butyl(1-hydroxyethyl)(phenyl)phosphine-borane $\left(S_{\mathrm{P}}-\mathbf{1}\right)$ was obtained from $\left(R_{\mathrm{P}}\right)$-O-adamantyl H-phosphinate (nearly $1: 1$ mixture of diastereomers) as a white solid.

Major Diastereoisomer (Like): ${ }^{1} \mathrm{H}$ NMR (400 $\left.\mathrm{MHz}, \mathrm{CDCl}_{3}\right) \delta$ $7.92(\mathrm{~m}, 2 \mathrm{H}), 7.50(\mathrm{~m}, 1 \mathrm{H}), 7.43(\mathrm{~m}, 2 \mathrm{H}),(\mathrm{q}, J=6.4 \mathrm{~Hz}, 1 \mathrm{H}), 1.85$ (br s, $1 \mathrm{H},-\mathrm{OH}), 1.52(\mathrm{dd}, J=6.8 \mathrm{~Hz}, J=14.0 \mathrm{~Hz}, 3 \mathrm{H}), 1.20(\mathrm{~d}, J=$ $13.6 \mathrm{~Hz}, 9 \mathrm{H}), 0.60\left(\mathrm{~m}, 3 \mathrm{H}, \mathrm{BH}_{3}\right) ;{ }^{31} \mathrm{P}\left\{{ }^{1} \mathrm{H}\right\} \mathrm{NMR}\left(162 \mathrm{MHz}, \mathrm{CDCl}_{3}\right)$ $\delta 38.85(\mathrm{~m}) ;{ }^{13} \mathrm{C}\left\{{ }^{1} \mathrm{H}\right\}$ NMR $\left(101 \mathrm{MHz}, \mathrm{CDCl}_{3}\right) \delta 134.91\left(\mathrm{~d}, J_{\mathrm{C}-\mathrm{P}}=\right.$ $7.3 \mathrm{~Hz}), 131.57\left(\mathrm{~d}, J_{\mathrm{C}-\mathrm{P}}=1.8 \mathrm{~Hz}\right), 128.43\left(\mathrm{~d}, J_{\mathrm{C}-\mathrm{P}}=9.2 \mathrm{~Hz}\right), 125.07$ $\left(\mathrm{d}, J_{\mathrm{C}-\mathrm{P}}=48.1 \mathrm{~Hz}\right), 65.20\left(\mathrm{~d}, J_{\mathrm{C}-\mathrm{P}}=36.8 \mathrm{~Hz}\right), 30.40\left(\mathrm{~d}, J_{\mathrm{C}-\mathrm{P}}=29.7\right.$ $\mathrm{Hz}), 26.91,20.80\left(\mathrm{~d}, J_{\mathrm{C}-\mathrm{P}}=4.8 \mathrm{~Hz}\right)$. Minor diastereoisomer (unlike): ${ }^{1} \mathrm{H}$ NMR $\left(400 \mathrm{MHz}, \mathrm{CDCl}_{3}\right) \delta 7.63(\mathrm{~m}, 2 \mathrm{H}), 7.52-7.48(\mathrm{~m}, 1 \mathrm{H})$, $7.45-7.39(\mathrm{~m}, 2 \mathrm{H}), 4.84(\mathrm{~m}, 1 \mathrm{H}), 2.15(\mathrm{br} \mathrm{d}, J=11.2 \mathrm{~Hz}, 1 \mathrm{H}$, $-\mathrm{OH}), 1.29(\mathrm{dd}, J=6.8 \mathrm{~Hz}, J=13.2 \mathrm{~Hz}, 3 \mathrm{H}), 1.18(\mathrm{~d}, J=13.6 \mathrm{~Hz}$, 9H), $0.62\left(\mathrm{~m}, 3 \mathrm{H}, \mathrm{BH}_{3}\right) ;{ }^{31} \mathrm{P}\left\{{ }^{1} \mathrm{H}\right\}$ NMR $\left(162 \mathrm{MHz} \mathrm{CDCl}_{3}\right) \delta 36.70$ $(\mathrm{m}) ;{ }^{13} \mathrm{C}\left\{{ }^{1} \mathrm{H}\right\} \operatorname{NMR}\left(101 \mathrm{MHz}, \mathrm{CDCl}_{3}\right) \delta 133.81\left(\mathrm{~d}, J_{\mathrm{C}-\mathrm{P}}=7.3 \mathrm{~Hz}\right)$, $131.65\left(\mathrm{~d}, J_{\mathrm{C}-\mathrm{P}}=2.2 \mathrm{~Hz}\right), 128.66\left(\mathrm{~d}, J_{\mathrm{C}-\mathrm{P}}=9.6 \mathrm{~Hz}\right), 126.51\left(\mathrm{~d}, J_{\mathrm{C}-\mathrm{P}}\right.$ $=47.0 \mathrm{~Hz}), 62.90\left(\mathrm{~d}, J_{\mathrm{C}-\mathrm{P}}=39.6 \mathrm{~Hz}\right), 30.08\left(\mathrm{~d}, J_{\mathrm{C}-\mathrm{P}}=30.1 \mathrm{~Hz}\right)$, $26.51\left(\mathrm{~d}, J_{\mathrm{C}-\mathrm{P}}=2.2 \mathrm{~Hz}\right), 20.23\left(\mathrm{~d}, J_{\mathrm{C}-\mathrm{P}}=6.0 \mathrm{~Hz}\right) ;{ }^{11} \mathrm{~B}$ NMR $(128$ $\left.\mathrm{MHz}, \mathrm{CDCl}_{3}\right) \delta-45.61\left(\mathrm{~d}, J_{\mathrm{B}-\mathrm{P}}=58.4 \mathrm{~Hz}\right) ;$ HPLC separation $((S, S)$-Whelk-O1 heptane/isopropanol 95:5 $1 \mathrm{~mL} / \mathrm{min}$ UV $254 \mathrm{~nm})$; $t_{\mathrm{R}}\left(R_{\mathrm{P}}, S\right)=5.97 \min ($ minor $), t_{\mathrm{R}}\left(S_{\mathrm{P}}, R\right)=6.64 \min ($ major $), t_{\mathrm{R}}\left(S_{\mathrm{P}}, S\right)$ $=7.80$ min $($ major $), t_{\mathrm{R}}\left(R_{\mathrm{P}}, R\right)=10.33 \mathrm{~min}$ (minor), er $=95: 5$ (major dia), 95:5 (minor dia).

$\left(R_{p}\right)$-tert-Butyl(1-hydroxyethyl)(phenyl)phosphine-borane $\left(R_{p}-1\right)$. HPLC separation $((S, S)$-Whelk-O1 heptane/isopropanol 95:5 $1 \mathrm{~mL} /$ $\min$ UV $254 \mathrm{~nm}$ ); $t_{\mathrm{R}}\left(R_{\mathrm{P}}, S\right)=6.27 \mathrm{~min}$ (major), $t_{\mathrm{R}}(S, R)=7.05 \mathrm{~min}$ (minor), $t_{\mathrm{R}}\left(S_{\mathrm{P}}, S\right)=8.33 \min ($ minor $), t_{\mathrm{R}}\left(R_{\mathrm{P}}, R\right)=10.99 \mathrm{~min}$ (major), er = 95:5 (major dia), 95.5:4.5 (minor dia).

General Procedure for the Umpolung Substitution of $\alpha$ Hydroxy Phosphine-Boranes. To a flame-dried $10 \mathrm{~mL}$ Schlenk tube was introduced under argon phosphine-borane $1(100 \mathrm{mg}, 0.446$ $\mathrm{mmol})$ dissolved in dry THF $(1 \mathrm{~mL})$, and the mixture was cooled to 0 ${ }^{\circ} \mathrm{C}$. At this temperature, the base ( 2 equiv) was added and the yellow mixture was stirred for an additional $5 \mathrm{~min}$ at $0{ }^{\circ} \mathrm{C}$. Then, the $\mathrm{N}$ iodosuccinimide ( 1 equiv) was added at $0{ }^{\circ} \mathrm{C}$, and the reaction was stirred for an additional $10 \mathrm{~min}$ at the same temperature. Finally, the nucleophile $\mathrm{NuH}$ ( 1 equiv) was added slowly at $0{ }^{\circ} \mathrm{C}$, and the reaction was allowed to warm to room temperature over $1 \mathrm{~h}$. After completion of the reaction (monitored by TLC), the reaction mixture was quenched with $2 \mathrm{~mL}$ of $0.35 \mathrm{M}$ aqueous $\mathrm{Na}_{2} \mathrm{~S}_{2} \mathrm{O}_{3}$ and $1 \mathrm{~mL}$ of saturated $\mathrm{NaHCO}_{3}$ and extracted 3 times with $5 \mathrm{~mL}$ of ethyl acetate. The organic layers were dried over $\mathrm{Na}_{2} \mathrm{SO}_{4}$, filtered, and concentrated under reduced pressure. The crude was then purified by column chromatography on silica gel using a combination of petroleum ether and ethyl acetate to yield the pure desired products.

$\left(R_{p}\right)$-N-Benzyl-1-phenyl-1-(tert-butyl)phosphinamine-borane $(2 a, M W=285.2 \mathrm{~g} / \mathrm{mol})$. This compound was obtained according to the general procedure from benzylamine as a white solid in $71 \%$ yield: mp 115.9-116.0 ${ }^{\circ} \mathrm{C} ;{ }^{1} \mathrm{H}$ NMR $\left(400 \mathrm{MHz} \mathrm{CDCl}_{3}\right) \delta 7.63(\mathrm{~m}, 2 \mathrm{H})$, $7.42(\mathrm{~m}, 4 \mathrm{H}), 7.26(\mathrm{~m}, 1 \mathrm{H}), 7.06(\mathrm{~m}, 2 \mathrm{H}), 4.33$ (dd, ABX system, $1 \mathrm{H}), 4.21$ (dd, ABX system, $1 \mathrm{H}), 2.18$ (bs, $1 \mathrm{H}, \mathrm{NH}), 1.10$ (d, $J=$ $14.23 \mathrm{~Hz}, 9 \mathrm{H}) ;{ }^{31} \mathrm{P}\left\{{ }^{1} \mathrm{H}\right\} \mathrm{NMR}\left(162 \mathrm{MHz} \mathrm{CDCl}_{3}\right) \delta 71.30$ (m, 1P); ${ }^{13} \mathrm{C}\left\{{ }^{1} \mathrm{H}\right\}$ NMR $\left(101 \mathrm{MHz} \mathrm{CDCl}_{3}\right) \delta 140.6\left(\mathrm{~d}, J_{\mathrm{P}-\mathrm{C}}=7.9 \mathrm{~Hz}\right), 131.9$ $\left(\mathrm{d}, J_{\mathrm{P}-\mathrm{C}}=9.3 \mathrm{~Hz}\right), 130.8\left(\mathrm{~d}, J_{\mathrm{P}-\mathrm{C}}=2.5 \mathrm{~Hz}\right), 130.6\left(\mathrm{~d}, J_{\mathrm{P}-\mathrm{C}}=46.5\right.$ $\mathrm{Hz}), 128.6,128.1\left(\mathrm{~d}, J_{\mathrm{P}-\mathrm{C}}=9.5 \mathrm{~Hz}\right), 127.9,127.4,47.1\left(\mathrm{~d}, J_{\mathrm{P}-\mathrm{C}}=1.7\right.$ $\mathrm{Hz}), 31.0\left(\mathrm{~d}, J_{\mathrm{P}-\mathrm{C}}=42.7 \mathrm{~Hz}\right), 24.7\left(\mathrm{~d}, J_{\mathrm{P}-\mathrm{C}}=2.8 \mathrm{~Hz}\right)$; IR $($ ATR $)$ $3348,3057,3028,2975,2963,2910,2867,2386,2372,2346,2281$, $1603,1492,1469,1391,1361,1297,1194,1156,1136,1111,1024$, 981, 937, 894, 814, 776, 741, 692, 643, 592, $507 \mathrm{~cm}^{-1}$; HRMS (ESITOF) $m / z[\mathrm{M}+\mathrm{Na}]^{+}$calcd for $\mathrm{C}_{17} \mathrm{H}_{25}$ BNPNa 308.1713, found 308.1714; $R_{f} 0.5$ (10:1 petroleum ether/AcOEt); HPLC separation (Chiralpak IB, heptane/ethanol (95:5), $1 \mathrm{~mL} / \mathrm{min}, \mathrm{UV} 220 \mathrm{~nm}) t_{\mathrm{R}}$ $\left(S_{\mathrm{P}}\right)=5.99 \mathrm{~min}$ (minor), $t_{\mathrm{R}}\left(R_{\mathrm{P}}\right)=6.50 \mathrm{~min}$ (major), er = 7.5:92.5, $[\alpha]_{\mathrm{D}}^{25}+25\left(c 0.2, \mathrm{CH}_{2} \mathrm{Cl}_{2}\right)$.

$\left(R_{P}\right)$-N-o-Fluorobenzyl-1-phenyl-1-(tert-butyl)phosphinamineborane $(\mathbf{2 b}, M W=303 \mathrm{~g} / \mathrm{mol})$. This compound was obtained according to the general procedure from $o$-fluorobenzylamine as a white solid in $76 \%$ yield: $\mathrm{mp} 88.7-90.1{ }^{\circ} \mathrm{C}$; ${ }^{1} \mathrm{H}$ NMR $(400 \mathrm{MHz}$ $\left.\mathrm{CDCl}_{3}\right) \delta 7.63(\mathrm{~m}, 2 \mathrm{H}), 7.42(\mathrm{~m}, 4 \mathrm{H}), 7.26(\mathrm{~m}, 1 \mathrm{H}), 7.06(\mathrm{~m}, 2 \mathrm{H})$, 4.33 (dd, ABX system, $1 \mathrm{H}), 4.21$ (dd, ABX system, $1 \mathrm{H}), 2.18$ (bs, $1 \mathrm{H}$, $\mathrm{NH}), 1.10(\mathrm{~d}, J=14.23 \mathrm{~Hz}, 9 \mathrm{H}), 1.04-0.5\left(\mathrm{t}, 3 \mathrm{H}, \mathrm{B} H_{3}\right) ;{ }^{31} \mathrm{P}\left\{{ }^{1} \mathrm{H}\right\}$ NMR (162 MHz CDCl $) \delta 71.40(\mathrm{~m}, 1 \mathrm{P}) ;{ }^{13} \mathrm{C}\left\{{ }^{1} \mathrm{H}\right\}$ NMR $(101 \mathrm{MHz}$ $\left.\mathrm{CDCl}_{3}\right) \delta 161.11\left(\mathrm{~d}, J_{\mathrm{F}-\mathrm{C}}=245 \mathrm{~Hz}\right), 131.8\left(\mathrm{~d}, J_{\mathrm{P}-\mathrm{C}}=9.35 \mathrm{~Hz}\right), 130.8$ $\left(\mathrm{d}, J_{\mathrm{P}-\mathrm{C}}=2.53 \mathrm{~Hz}\right), 130.5\left(\mathrm{~d}, J_{\mathrm{F}-\mathrm{C}}=4.61 \mathrm{~Hz}\right), 129.1\left(\mathrm{~d}, J_{\mathrm{F}-\mathrm{C}}=8.19\right.$ $\mathrm{Hz}), 128.0\left(\mathrm{~d}, J_{\mathrm{P}-\mathrm{C}}=9.53 \mathrm{~Hz}\right), 127.6\left(\mathrm{~d}, J_{\mathrm{C}-\mathrm{F}}=6.38 \mathrm{~Hz}\right), 127.4(\mathrm{~d}$, $\left.J_{\mathrm{C}-\mathrm{F}}=6.33 \mathrm{~Hz}\right), 124.2\left(\mathrm{~d}, J_{\mathrm{C}-\mathrm{F}}=3.49 \mathrm{~Hz}\right), 41.1(\mathrm{~m}), 30.94\left(\mathrm{~d}, J_{\mathrm{P}-\mathrm{C}}=\right.$ $40 \mathrm{~Hz}), 24.63\left(\mathrm{~d}, J_{\mathrm{P}-\mathrm{C}}=2.85 \mathrm{~Hz}\right) ;{ }^{11} \mathrm{~B}$ NMR $\left(128 \mathrm{MHz}^{\mathrm{CDCl}}{ }_{3}\right) \delta$ -46.26; IR (ATR) 3337, 2960, 2941, 9865, 9835, 2383, 1602, 1491, $1462,1423,1303,1239,1160,1109,1049,999,924,856,811,760$, $735,694,641,618,600,564$; HRMS (ESI-TOF) $m / z[\mathrm{M}+\mathrm{Na}]^{+}$ calcd for $\mathrm{C}_{17} \mathrm{H}_{24}$ BFNPNa 326.1619, found 326.1622; $R_{f} 0.4$ (9:1 petroleum ether/AcOEt); HPLC separation (Lux-Cellulose-2, heptane/isopropanol (95:5), $1 \mathrm{~mL} / \mathrm{min}, 254 \mathrm{~nm}), t_{\mathrm{R}}$ first eluted $6.86 \mathrm{~min}$ (major), $t_{\mathrm{R}}$ second eluted $9.18 \mathrm{~min}$ (minor), er $=$ 94:6 
$\left(R_{p}\right)$-N-o-Methoxybenzyl-1-phenyl-1-(tert-butyl)phosphinamineborane $(2 \mathrm{c}, M W=315 \mathrm{~g} / \mathrm{mol})$. This compound was obtained according to the general procedure from $o$-methoxybenzylamine as a white solid in $63 \%$ yield: $\mathrm{mp} 90.3-90.4{ }^{\circ} \mathrm{C}$; ${ }^{1} \mathrm{H}$ NMR $(400 \mathrm{MHz}$ $\left.\mathrm{CDCl}_{3}\right) \delta 7.64(\mathrm{~m}, 2 \mathrm{H}), 7.40(\mathrm{~m}, 3 \mathrm{H}), 7.25(\mathrm{~m}, 2 \mathrm{H}), 6.86(\mathrm{~m}, 2 \mathrm{H})$, $4.24(\mathrm{dd}, \mathrm{ABX}$ system, $1 \mathrm{H}), 4.16(\mathrm{dd}, \mathrm{ABX}$ system, $1 \mathrm{H}), 3.88(\mathrm{~s}, 3 \mathrm{H})$, 2.5 (bs, $1 \mathrm{H}, \mathrm{NH}), 1.08$ (d, $J=14.08 \mathrm{~Hz}, 9 \mathrm{H}), 0.91-0.5\left(\mathrm{t}, 3 \mathrm{H}, \mathrm{BH}_{3}\right)$; ${ }^{31} \mathrm{P}\left\{{ }^{1} \mathrm{H}\right\}$ NMR $\left(162 \mathrm{MHz} \mathrm{CDCl}_{3}\right) \delta 70.30(\mathrm{~m}, 1 \mathrm{P}) ;{ }^{13} \mathrm{C}\left\{{ }^{1} \mathrm{H}\right\}$ NMR $\left(101 \mathrm{MHz} \mathrm{CDCl}_{3}\right) \delta 157.6(\mathrm{~s}), 131.9\left(\mathrm{~d}, J_{\mathrm{P}-\mathrm{C}}=9.35 \mathrm{~Hz}\right), 130.8(\mathrm{~d}$, $\left.J_{\mathrm{P}-\mathrm{C}}=2.53 \mathrm{~Hz}\right), 130.5\left(\mathrm{~d}, J_{\mathrm{P}-\mathrm{C}}=2.53 \mathrm{~Hz}\right), 129.8(\mathrm{~s}), 128.7(\mathrm{~s}), 127.9$ $\left(\mathrm{d}, J_{\mathrm{C}-\mathrm{P}}=9.43 \mathrm{~Hz}\right), 120.7(\mathrm{~s}), 110.3(\mathrm{~s}), 55.28(\mathrm{~s}), 43.2\left(\mathrm{~d}, J_{\mathrm{P}-\mathrm{C}}=2\right.$ $\mathrm{Hz}), 30.7\left(\mathrm{~d}, J_{\mathrm{P}-\mathrm{C}}=45 \mathrm{~Hz}\right), 24.7\left(\mathrm{~d}, J_{\mathrm{P}-\mathrm{C}}=2.78 \mathrm{~Hz}\right) ;{ }^{11} \mathrm{~B}$ NMR $(128$ $\mathrm{MHz} \mathrm{CDCl}_{3}$ ) $\delta$-42.22; IR (ATR) 3351, 3328, 3060, 2966, 2913, $1867,2343,2265,1585,1485,1433,1421,1363,1330,1293,1177$, $1076,1014,998,862,848,814,774,755,695,645,573,519$; HRMS (ESI-TOF) $\mathrm{m} / z[\mathrm{M}+\mathrm{Na}]^{+}$calcd for $\mathrm{C}_{18} \mathrm{H}_{27} \mathrm{BNOPNa} 338.1819$ found 338.1816; $R_{f} 0.3$ (9:1 petroleum ether/AcOEt); HPLC separation (Chiralcel OD-3, heptane/isopropanol (95:5), $1 \mathrm{~mL}$ / $\min , 220 \mathrm{~nm}$ ), $t_{\mathrm{R}}$ first eluted $6.061 \mathrm{~min}$ (minor), $t_{\mathrm{R}}$ second eluted 7.446 min (major), er $=7: 93$

$\left(R_{p}\right)$-N-o-Chlorobenzyl-1-phenyl-1-(tert-butyl)phosphinamineborane $(2 d, M W=319 \mathrm{~g} / \mathrm{mol})$. This compound was obtained according to the general procedure from $o$-chlorobenzylamine as a white solid in $72 \%$ yield: $\mathrm{mp} 81.3-90.6{ }^{\circ} \mathrm{C}$; ${ }^{1} \mathrm{H}$ NMR $(400 \mathrm{MHz}$ $\left.\mathrm{CDCl}_{3}\right) \delta 7.61(\mathrm{~m}, 2 \mathrm{H}), 7.40(\mathrm{~m}, 5 \mathrm{H}), 7.17(\mathrm{~m}, 2 \mathrm{H}), 4.37$ (dd, $\mathrm{ABX}$ system, $1 \mathrm{H}), 4.26(\mathrm{dd}, \mathrm{ABX}$ system, $1 \mathrm{H}), 2.59$ (bs, $1 \mathrm{H}, \mathrm{NH}), 1.09$ (d, $J=14.12 \mathrm{~Hz}, 9 \mathrm{H}), 0.91-0.5\left(\mathrm{t}, 3 \mathrm{H}, \mathrm{BH}{ }_{3}\right) ;{ }^{31} \mathrm{P}\left\{{ }^{1} \mathrm{H}\right\} \mathrm{NMR}(162 \mathrm{MHz}$ $\left.\mathrm{CDCl}_{3}\right) \delta 71.15(\mathrm{~m}, 1 \mathrm{P}) ;{ }^{13} \mathrm{C}\left\{{ }^{1} \mathrm{H}\right\} \operatorname{NMR}\left(101 \mathrm{MHz} \mathrm{CDCl}_{3}\right) \delta 137.9$ $\left(\mathrm{d}, J_{\mathrm{P}-\mathrm{H}}=5.82 \mathrm{~Hz}\right), 133.5(\mathrm{~s}), 131.7\left(\mathrm{~d}, J_{\mathrm{P}-\mathrm{C}}=9.35 \mathrm{~Hz}\right), 130.7(\mathrm{~s})$, $130.7\left(\mathrm{~d}, J_{\mathrm{P}-\mathrm{C}}=2.53 \mathrm{~Hz}\right), 130.5\left(\mathrm{~d}, J_{\mathrm{P}-\mathrm{C}}=2.53 \mathrm{~Hz}\right), 129.4(\mathrm{~s}), 128.8$ $(\mathrm{s}), 128.0\left(\mathrm{~d}, J_{\mathrm{C}-\mathrm{P}}=9.51 \mathrm{~Hz}\right), 127.1(\mathrm{~s}), 45.0\left(\mathrm{~d}, J_{\mathrm{P}-\mathrm{C}}=2,49 \mathrm{~Hz}\right)$, $30.9\left(\mathrm{~d}, J_{\mathrm{P}-\mathrm{C}}=45 \mathrm{~Hz}\right), 24.6\left(\mathrm{~d}, J_{\mathrm{P}-\mathrm{C}}=2.84 \mathrm{~Hz}\right) ;{ }^{11} \mathrm{~B}$ NMR $(128 \mathrm{MHz}$ $\mathrm{CDCl}_{3}$ ) $\delta$-42.3; IR (ATR) 3384, 3328, 3061, 2963, 2930, 2902, $1384,2340,1588,1572,1434,1405,1363,1328,1261,1244,1126$, $1091,1067,1047,1014,1000,940,912,891,867,815,754,739,701$, 612, 599; HRMS (ESI-TOF) $m / z[\mathrm{M}+\mathrm{Na}]^{+}$calcd for $\mathrm{C}_{17} \mathrm{H}_{24} \mathrm{BClNPNa} 342.1323$, found $342.1321 ; R_{f} 0.4$ (9:1 petroleum ether/AcOEt); HPLC separation (Chiralpak AS-H, heptane/ isopropanol $(95: 5), 1 \mathrm{~mL} / \mathrm{min}, 220 \mathrm{~nm}), t_{\mathrm{R}}$ first eluted $4.52 \mathrm{~min}$ (major), $t_{\mathrm{R}}$ second eluted $5.32 \mathrm{~min}$ (minor), er $=89: 11$

$\left(R_{p}\right)$-N-m-Methoxybenzyl-1-phenyl-1-(tert-butyl)phosphinamine-borane $(2 e, M W=315 \mathrm{~g} / \mathrm{mol})$. This compound was obtained according to the general procedure from $m$ methoxybenzylamine as a white solid in $76 \%$ yield: $\mathrm{mp}$ 63.9-64.0 ${ }^{\circ} \mathrm{C} ;{ }^{1} \mathrm{H}$ NMR $\left(400 \mathrm{MHz}^{\circ} \mathrm{CDCl}_{3}\right) \delta 7.68(\mathrm{~m}, 2 \mathrm{H}), 7.45(\mathrm{~m}, 3 \mathrm{H}), 7.24$ $(\mathrm{m}, 1 \mathrm{H}), 6.92(\mathrm{~m}, 2 \mathrm{H}), 6.82(\mathrm{~m}, 1 \mathrm{H}) 4.29(\mathrm{dd}, \mathrm{ABX}$ system, $1 \mathrm{H})$, 4.09 (dd, ABX system, $1 \mathrm{H}), 3.76(\mathrm{~s}, 3 \mathrm{H}), 2.02(\mathrm{bs}, 1 \mathrm{H}, \mathrm{NH}), 1.08(\mathrm{~d}$, $J=14.08 \mathrm{~Hz}, 9 \mathrm{H}), 0.91-0.5(\mathrm{t}, 3 \mathrm{H}, \mathrm{BH}) ;{ }^{31} \mathrm{P}\left\{{ }^{1} \mathrm{H}\right\} \operatorname{NMR}(162 \mathrm{MHz}$ $\left.\mathrm{CDCl}_{3}\right) \delta 71.12(\mathrm{~m}, 1 \mathrm{P}) ;{ }^{13} \mathrm{C}\left\{{ }^{1} \mathrm{H}\right\} \mathrm{NMR}\left(101 \mathrm{MHz}^{\mathrm{CDCl}}{ }_{3}\right) \delta 157.6$ $(\mathrm{s}), 142.3\left(\mathrm{~d}, J_{\mathrm{P}-\mathrm{H}}=7.41 \mathrm{~Hz}\right), 131.9\left(\mathrm{~d}, J_{\mathrm{P}-\mathrm{C}}=9.35 \mathrm{~Hz}\right), 130.8(\mathrm{~d}$, $\left.J_{\mathrm{P}-\mathrm{C}}=2.53 \mathrm{~Hz}\right), 129.8(\mathrm{~s}), 129.6(\mathrm{~s}), 128.12\left(\mathrm{~d}, J_{\mathrm{C}-\mathrm{P}}=9.50 \mathrm{~Hz}\right)$ $120.3(\mathrm{~s}), 113.2\left(\mathrm{~d}, J_{\mathrm{P}-\mathrm{H}}=12.62 \mathrm{~Hz}\right), 55.16(\mathrm{~s}), 47.7\left(\mathrm{~d}, J_{\mathrm{P}-\mathrm{C}}=2\right.$ $\mathrm{Hz}), 30.7\left(\mathrm{~d}, J_{\mathrm{P}-\mathrm{C}}=45.3 \mathrm{~Hz}\right), 24.7\left(\mathrm{~d}, J_{\mathrm{P}-\mathrm{C}}=2.82 \mathrm{~Hz}\right) ;{ }^{11} \mathrm{~B} \mathrm{NMR}$ $\left(128 \mathrm{MHz} \mathrm{CDCl}_{3}\right.$ ) $\delta-42,15$; IR (ATR) 3347, 3334, 3053, 2959, 2918, 1834, 2378, 2354, 1609, 1584, 1454, 1434, 1221, 1189, 1154 $1077,1016,999,940,882,829,817,761,649,574,538$; HRMS (ESITOF) $\mathrm{m} / z[\mathrm{M}+\mathrm{Na}]^{+}$calcd for $\mathrm{C}_{18} \mathrm{H}_{27}$ BNOPNa 338.1819, found 338.1820; $R_{f} 0.3$ (9:1 petroleum ether/AcOEt) HPLC separation (Chiralpak AS-H, heptane/isopropanol (95:5), $1 \mathrm{~mL} / \mathrm{min}, 230 \mathrm{~nm}$ ), $t_{\mathrm{R}}$ first eluted $6.546 \mathrm{~min}$ (major), $t_{\mathrm{R}}$ second eluted $7.99 \mathrm{~min}$ (minor), er $=87.5: 12.5 ;[\alpha]_{\mathrm{D}}^{25}+26\left(c 0.19, \mathrm{CH}_{2} \mathrm{Cl}_{2}\right)$

$\left(R_{p}\right)$-N-p-Methoxybenzyl-1-phenyl-1-(tert-butyl)phosphinamineborane $(2 f, M W=315 \mathrm{~g} / \mathrm{mol})$. This compound was obtained according to the general procedure from $p$-methoxybenzylamine as a white solid in $81 \%$ yield: $\mathrm{mp}$ 91.5-92.4 ${ }^{\circ} \mathrm{C}$; ${ }^{1} \mathrm{H}$ NMR $(400 \mathrm{MHz}$ $\left.\mathrm{CDCl}_{3}\right) \delta 7.77(\mathrm{~m}, 2 \mathrm{H}), 7.46(\mathrm{~m}, 3 \mathrm{H}), 6.88(\mathrm{~m}, 2 \mathrm{H}), 6.73(\mathrm{~m}, 2 \mathrm{H})$, 4.29 (dd, ABX system, 1H), 4.09 (dd, ABX system, $1 \mathrm{H}), 3.72(\mathrm{~s}, 3 \mathrm{H})$, 1.56 (bs, $1 \mathrm{H}, \mathrm{NH}), 1.22(\mathrm{~d}, J=14.08 \mathrm{~Hz}, 9 \mathrm{H}), 0.91-0.7(\mathrm{t}, 3 \mathrm{H}$, $\left.\mathrm{BH}_{3}\right) ;{ }^{31} \mathrm{P}\left\{{ }^{1} \mathrm{H}\right\} \operatorname{NMR}\left(162 \mathrm{MHz} \mathrm{CDCl}_{3}\right) \delta 70.49(\mathrm{~m}, 1 \mathrm{P}) ;{ }^{13} \mathrm{C}\left\{{ }^{1} \mathrm{H}\right\}$ NMR (101 MHz $\left.\mathrm{CDCl}_{3}\right) \delta 159.0(\mathrm{~s}), 132.8\left(\mathrm{~d}, J_{\mathrm{P}-\mathrm{H}}=7.87 \mathrm{~Hz}\right)$, $132.0\left(\mathrm{~d}, J_{\mathrm{P}-\mathrm{C}}=9.31 \mathrm{~Hz}\right), 130.8\left(\mathrm{~d}, J_{\mathrm{P}-\mathrm{C}}=2.53 \mathrm{~Hz}\right), 129.2(\mathrm{~s}), 129.6$ $(\mathrm{s}), 128.1\left(\mathrm{~d}, J_{\mathrm{C}-\mathrm{P}}=9.45 \mathrm{~Hz}\right), 114.0(\mathrm{~s}), 55.31(\mathrm{~s}), 46 .\left(\mathrm{d}, J_{\mathrm{P}-\mathrm{C}}=1.82\right.$ $\mathrm{Hz}), 31.2\left(\mathrm{~d}, J_{\mathrm{P}-\mathrm{C}}=45.3 \mathrm{~Hz}\right), 24.7\left(\mathrm{~d}, J_{\mathrm{P}-\mathrm{C}}=2.81 \mathrm{~Hz}\right) ;{ }^{11} \mathrm{~B}$ NMR $\left(128 \mathrm{MHz} \mathrm{CDCl}_{3}\right.$ ) $\delta$-42,09; IR (ATR) 3330, 3055, 3000, 2969, $2967,2407,2378,2354,1611,1512,1460,1434,1392,1363,1302$, $1250,1212,1103,1077,1026,1014,974,866,821,760,738,701$, $648,616,596,552,518$; HRMS (ESI-TOF) $m / z[\mathrm{M}+\mathrm{Na}]^{+}$calcd for $\mathrm{C}_{18} \mathrm{H}_{27} \mathrm{BNOPNa} 338.1819$, found 338.1816; $R_{f}=0.2(10: 1$ petroleum ether/AcOEt); HPLC separation (Lux cellulose 4, heptane/ isopropanol $(95: 5), 1 \mathrm{~mL} / \mathrm{min}, 220 \mathrm{~nm}), t_{\mathrm{R}}$ first eluted $7.78 \mathrm{~min}$ (minor), $t_{\mathrm{R}}$ second eluted 8.45 min (major), er $=5.5: 94.5$

$\left(R_{P}\right)$-N-Pentyl-1-phenyl-1-(tert-butyl)phosphinamine-borane $(2 \mathrm{~g}, M W=365 \mathrm{~g} / \mathrm{mol})$. This compound was obtained according to the general procedure from pentylamine as a white solid in $43 \%$ yield: $\mathrm{mp}$ 65.0-65.1 ${ }^{\circ} \mathrm{C} ;{ }^{1} \mathrm{H} \mathrm{NMR}\left(400 \mathrm{MHz} \mathrm{CDCl}_{3}\right) \delta 7.68(\mathrm{~m}, 2 \mathrm{H}), 7.45$ $(\mathrm{m}, 3 \mathrm{H}), 3.07(\mathrm{~m}, 1 \mathrm{H}), 2.94(\mathrm{~m}, 1 \mathrm{H}), 1.70(\mathrm{bs}, 1 \mathrm{H}), 1.52(\mathrm{~m}, 2 \mathrm{H})$, $1.31(\mathrm{~m}, 4 \mathrm{H}), 1.09(\mathrm{~d}, J=14 \mathrm{~Hz}, 9 \mathrm{H}), 0.89(\operatorname{app~t}, 3 \mathrm{H}, J=6.83 \mathrm{~Hz})$, $0.8-0.4\left(\mathrm{t}, 3 \mathrm{H}, \mathrm{BH}_{3}\right) ;{ }^{31} \mathrm{P}\left\{{ }^{1} \mathrm{H}\right\}$ NMR $\left(162 \mathrm{MHz} \mathrm{CDCl}_{3}\right) \delta 69.90(\mathrm{~m}$, $1 \mathrm{P}) ;{ }^{13} \mathrm{C}\left\{{ }^{1} \mathrm{H}\right\}$ NMR (101 MHz CDCl $\left.{ }_{3}\right) \delta 131.9\left(\mathrm{~d}, J_{\mathrm{P}-\mathrm{H}}=9.25 \mathrm{~Hz}\right)$, $130.7\left(\mathrm{~d}, J_{\mathrm{P}-\mathrm{C}}=2.51 \mathrm{~Hz}\right), 128.0\left(\mathrm{~d}, J_{\mathrm{C}-\mathrm{P}}=9.45 \mathrm{~Hz}\right), 43.1(\mathrm{~s}), 32.31$ $\left(\mathrm{d}, J_{\mathrm{P}-\mathrm{H}}=5.95 \mathrm{~Hz}\right), 30.9\left(\mathrm{~d}, J_{\mathrm{P}-\mathrm{C}}=45.3 \mathrm{~Hz}\right), 24.7\left(\mathrm{~d}, J_{\mathrm{P}-\mathrm{C}}=2.76\right.$ $\mathrm{Hz}), 22.38(\mathrm{~s}), 14.0(\mathrm{~s}) ;{ }^{11} \mathrm{~B}$ NMR $\left(128 \mathrm{MHz}^{\mathrm{CDCl}}{ }_{3}\right) \delta-42,29$; IR (ATR) 3361, 3057, 2960, 2928, 2867, 2369, 2343, 1467, 1434, 1409, $1390,1195,1136,1100,1069,1016,998,896,850,815,739,696$, 640, 573; HRMS (ESI-TOF) $m / z[\mathrm{M}+\mathrm{Na}]^{+}$calcd for $\mathrm{C}_{15} \mathrm{H}_{29} \mathrm{BNPNa} 288.2026$, found 288.2028; $R_{f}=0.9$ (9:1 petroleum ether/AcOEt); HPLC separation (Chiralpak IG, heptane/isopropanol $(95: 5), 1 \mathrm{~mL} / \mathrm{min}, 254 \mathrm{~nm}), t_{\mathrm{R}}$ first eluted $5.499 \mathrm{~min}$ (minor), $t_{\mathrm{R}}$ second eluted $6.199 \mathrm{~min}$ (major), er = 11.5:88.5; $[\alpha]_{\mathrm{D}}^{25}+45(c 0.198$, $\mathrm{CH}_{2} \mathrm{Cl}_{2}$ )

$\left(R_{P}\right)$-1-Amino-1-phenyl-1-(tert-butyl)phosphinamine-borane $(2 \mathrm{~h}, M W=195 \mathrm{~g} / \mathrm{mol})$. This compound was obtained according to the general procedure from ammonia $(0.4 \mathrm{M}$ in THF) as a white solid in $40 \%$ yield: $\mathrm{mp} 110.7-110.8{ }^{\circ} \mathrm{C}$; ${ }^{1} \mathrm{H}$ NMR $\left(400 \mathrm{MHz} \mathrm{CDCl}_{3}\right) \delta$ $7.70(\mathrm{~m}, 2 \mathrm{H}), 7.46(\mathrm{~m}, 3 \mathrm{H}), 1.99$ (bs, $\left.1 \mathrm{H}, \mathrm{NH}_{2}\right), 1.11$ (d, $J=14 \mathrm{~Hz}$, 9H), 0.89-0.50 (t, 3H, BH $\left.{ }_{3}\right) ;{ }^{31} \mathrm{P}\left\{{ }^{1} \mathrm{H}\right\}$ NMR $\left(162 \mathrm{MHz} \mathrm{CDCl}_{3}\right) \delta$ $63.58(\mathrm{~m}, 1 \mathrm{P}) ;{ }^{13} \mathrm{C}\left\{{ }^{1} \mathrm{H}\right\}$ NMR $\left(101 \mathrm{MHz}^{\mathrm{C} C \mathrm{CDl}_{3}}\right) \delta 131.6\left(\mathrm{~d}, J_{\mathrm{p}-\mathrm{H}}=\right.$ $9.78 \mathrm{~Hz}), 130.8\left(\mathrm{~d}, J_{\mathrm{P}-\mathrm{C}}=2.57 \mathrm{~Hz}\right), 128.1\left(\mathrm{~d}, J_{\mathrm{C}-\mathrm{P}}=9.87 \mathrm{~Hz}\right), 30.6$ $\left(\mathrm{d}, J_{\mathrm{P}-\mathrm{C}}=45.4 \mathrm{~Hz}\right), 24.4\left(\mathrm{~d}, J_{\mathrm{P}-\mathrm{C}}=3.29 \mathrm{~Hz}\right) ;{ }^{11} \mathrm{~B}$ NMR $(128 \mathrm{MHz}$ $\mathrm{CDCl}_{3}$ ) $\delta-40,50$; IR (ATR) 3424, 3333, 3076, 3054, 2978, 2965, 2942, 2926, 2899,2374, 2348, 2280, 1552, 1488, 1472, 1395, 1315, 1140, 1108, 1084, 1016, 967, 937, 901, 812, 740, 701, 692, 643, 617, 574; HPLC separation (Chiralcel OD-3, heptane/ethanol (80:20), 1 $\mathrm{mL} / \mathrm{min}, 260 \mathrm{~nm}$ ), $t_{\mathrm{R}}$ first eluted 4.956 min (major), $t_{\mathrm{R}}$ second eluted 6.340 min (minor), er $=81: 19 ;[\alpha]_{\mathrm{D}}^{25}+6\left(c 0.57, \mathrm{CH}_{2} \mathrm{Cl}_{2}\right)$. These data are consistent with those in the literature. ${ }^{5 a}$ 
(1) (a) Börner, A., Ed. Phosphorus Ligands in Asymmetric Catalysis; Synthesis and Applications; Wiley-VCH Verlag GmbH \& Co. KGaA, 2008. (b) Marinetti, A.; Voituriez, A. Enantioselective Phosphine Organocatalysis. Synlett 2010, 2010, 174-194. (c) Grabulosa, A., Ed. P-Stereogenic Ligands in Enantioselective Catalysis; Royal Society of Chemistry Publishing, 2011. (d) Dutartre, M.; Bayardon, J.; Jugé, S. Applications and Stereoselective Syntheses of P-Chirogenic Phosphorus Compounds. Chem. Soc. Rev. 2016, 45, 5771-5794. (e) Fu, W.; Tang, W. Chiral Monophosphorus Ligands for Asymmetric Catalytic Reactions. ACS Catal. 2016, 6, 4814-4858.

(2) (a) Jin, M.; Adak, L.; Nakamura, M. Iron-Catalyzed Enantioselective Cross-Coupling Reactions of $\alpha$-Chloroesters with Aryl Grignard Reagents. J. Am. Chem. Soc. 2015, 137, 7128-7134. (b) You, W.; Brown, M. K. Catalytic Enantioselective Diarylation of Alkenes. J. Am. Chem. Soc. 2015, 137, 14578-14581.

(3) (a) Alayrac, C.; Lakhdar, S.; Abdellah, I.; Gaumont, A.-C. Recent Advances in Synthesis of P-BH3 Compounds. In Phosphorus Chemistry II; Montchamp, J.-L., Ed.; Springer International Publishing: Cham, 2014; Vol. 361, pp 1-82. For recent examples, see: (b) Clavero, P.; Grabulosa, A.; Rocamora, M.; Muller, G.; FontBardia, M. Ruthenium Complexes of P-Stereogenic Phosphines with a Heterocyclic Substituent. Dalton Trans. 2016, 45, 8513-8531. (c) Kolodiazhnyi, O. I. Stereoselective Reactions of Trivalent Phosphorus Compounds. Russ. J. Gen. Chem. 2017, 87, 425-441. (d) Herrera, A.; Grasruck, A.; Heinemann, F. W.; Scheurer, A.; Chelouan, A.; Frieß, S.; Seidel, F.; Dorta, R. Developing PStereogenic, Planar-Chiral P-Alkene Ligands: Monodentate, Bidentate, and Double Agostic Coordination Modes on $\mathrm{Ru}(\mathrm{II})$. Organometallics 2017, 36, 714-720.

(4) For a review, see: (a) Shaikh, T. M.; Weng, C.-M.; Hong, F.-E. Secondary Phosphine Oxides: Versatile Ligands in Transition MetalCatalyzed Cross-Coupling Reactions. Coord. Chem. Rev. 2012, 256, 771-803 and references cited therein . (b) Clavier, H.; Buono, G. [2 +1 ] Cycloaddition Affording Methylene- and Vinylidenecyclopropane Derivatives: A Journey around the Reactivity of MetalPhosphinito-Phosphinous Acid Complexes. Chem. Rec. 2017, 17, 399-414. For recent examples, see: (c) Vasseur, A.; Membrat, R.; Palpacelli, D.; Giorgi, M.; Nuel, D.; Giordano, L.; Martinez, A. Synthesis of Chiral Supramolecular Bisphosphinite Palladacycles through Hydrogen Transfer-Promoted Self-Assembly Process. Chem. Commun. 2018, 54, 10132-10135. (d) Vasseur, A.; Membrat, R. Gatineau, D.; Tenaglia, A.; Nuel, D.; Giordano, L. Secondary Phosphine Oxides as Multitalented Preligands En Route to the Chemoselective Palladium-Catalyzed Oxidation of Alcohols. ChemCatChem 2017, 9, 728-732. (e) Membrat, R.; Vasseur, A.; Martinez, A.; Giordano, L.; Nuel, D. Phosphinous Acid Platinum Complex as Robust Catalyst for Oxidation: Comparison with Palladium and Mechanistic Investigations: Phosphinous Acid Platinum Complex as Robust Catalyst for Oxidation: Comparison with Palladium and Mechanistic Investigations. Eur. J. Org. Chem. 2018, 2018, 54275434. (f) Han, Z. S.; Wu, H.; Xu, Y.; Zhang, Y.; Qu, B.; Li, Z.; Caldwell, D. R.; Fandrick, K. R.; Zhang, L.; Roschangar, F.; Song, J. J.; Senanayake, C. H. General and Stereoselective Method for the Synthesis of Sterically Congested and Structurally Diverse $P$ -Stereogenic Secondary Phosphine Oxides. Org. Lett. 2017, 19, $1796-1799$

(5) (a) Revés, M.; Ferrer, C.; León, T.; Doran, S.; Etayo, P.; VidalFerran, A.; Riera, A.; Verdaguer, X. Primary and Secondary Aminophosphines as Novel P-Stereogenic Building Blocks for Ligand
Synthesis. Angew. Chem., Int. Ed. 2010, 49, 9452-9455. (b) León, T.; Parera, M.; Roglans, A.; Riera, A.; Verdaguer, X. P-Stereogenic Secondary Iminophosphorane Ligands and Their Rhodium(I) Complexes: Taking Advantage of $\mathrm{NH} / \mathrm{PH}$ Tautomerism. Angew. Chem., Int. Ed. 2012, 51, 6951-6955. (c) Flores-Gaspar, A.; Orgué, S.; Grabulosa, A.; Riera, A.; Verdaguer, X. Borane as an Efficient Directing Group. Stereoselective 1,2-Addition of Organometallic Reagents to Borane P-Stereogenic N-Phosphanylimines. Chem. Commun. 2015, 51, 1941-1944. (d) Kolodiazhnyi, O. I.; Kolodiazhna, A. Nucleophilic Substitution at Phosphorus: Stereochemistry and Mechanisms. Tetrahedron: Asymmetry 2017, 28, 16511674. (e) Téllez, J.; Gallen, A.; Ferrer, J.; Lahoz, F. J.; García-Orduña, P.; Riera, A.; Verdaguer, X.; Carmona, D.; Grabulosa, A. HalfSandwich Complexes of $\mathrm{Ir}(\mathrm{III}), \mathrm{Rh}(\mathrm{III})$ and $\mathrm{Ru}(\mathrm{II})$ with the MaxPhos Ligand: Metal Centred Chirality and Cyclometallation. Dalton Trans. 2017, 46, 15865-15874. (f) Salomó, E.; Rojo, P.; Hernández-Lladó, P.; Riera, A.; Verdaguer, X. P-Stereogenic and Non-P-Stereogenic IrMaxPHOX in the Asymmetric Hydrogenation of $N$-Aryl Imines. Isolation and X-Ray Analysis of Imine Iridacycles. J. Org. Chem. 2018, 83, 4618-4627. (g) Salomó, E.; Gallen, A.; Sciortino, G.; Ujaque, G.; Grabulosa, A.; Lledós, A.; Riera, A.; Verdaguer, X. Direct Asymmetric Hydrogenation of $N$-Methyl and $N$-Alkyl Imines with an $\operatorname{Ir}(\mathrm{III}) \mathrm{H}$ Catalyst. J. Am. Chem. Soc. 2018, 140, 16967-16970. (h) Kolodiazhnyi, O. I. Stereochemistry of Electrophilic and Nucleophilic Substitution at Phosphorus. Phosphorus, Sulfur Silicon Relat. Elem. 2018, 1-5.

(6) Cristóbal-Lecina, E.; Costantino, A. R.; Grabulosa, A.; Riera, A.; Verdaguer, X. Rhodium-Catalyzed Pauson-Khand Reaction Using a Small-Bite-Angle $P$-Stereogenic $C_{1}$-Diphosphine Ligand. Organometallics 2015, 34, 4989-4993.

(7) (a) Cristóbal-Lecina, E.; Etayo, P.; Doran, S.; Revés, M.; MartínGago, P.; Grabulosa, A.; Costantino, A. R.; Vidal-Ferran, A.; Riera, A.; Verdaguer, X. MaxPHOS Ligand: $\mathrm{PH} / \mathrm{NH}$ Tautomerism and Rhodium-Catalyzed Asymmetric Hydrogenations. Adv. Synth. Catal. 2014, 356, 795-804. (b) Salomó, E.; Orgué, S.; Riera, A.; Verdaguer, $\mathrm{X}$. Highly Enantioselective Iridium-Catalyzed Hydrogenation of Cyclic Enamides. Angew. Chem., Int. Ed. 2016, 55, 7988-7992.

(8) Gatineau, D.; Nguyen, D. H.; Hérault, D.; Vanthuyne, N.; Leclaire, J.; Giordano, L.; Buono, G. H-Adamantylphosphinates as Universal Precursors of P-Stereogenic Compounds. J. Org. Chem. 2015, 80, 4132-4141.

(9) (a) Juge, S.; Stephan, M.; Laffitte, J. A.; Genet, J. P. Efficient Asymmetric Synthesis of Optically Pure Tertiary Mono and Diphosphine Ligands. Tetrahedron Lett. 1990, 31, 6357-6360. (b) Imamoto, T.; Watanabe, J.; Wada, Y.; Masuda, H.; Yamada, H.; Tsuruta, H.; Matsukawa, S.; Yamaguchi, K. P-Chiral Bis (Trialkylphosphine) Ligands and Their Use in Highly Enantioselective Hydrogenation Reactions. J. Am. Chem. Soc. 1998, 120, 1635-1636. (c) Nagata, K.; Matsukawa, S.; Imamoto, T. Stereoselective, Oxidative One-Carbon Degradation of Alkyl(dimethyl)phosphine-Boranes. Synthesis of Enantiomerically Enriched Secondary PhosphineBoranes. J. Org. Chem. 2000, 65, 4185-4188. (d) Bergin, E.; O'Connor, C. T.; Robinson, S. B.; McGarrigle, E. M.; O’Mahony, C. P.; Gilheany, D. G. Synthesis of P-Stereogenic Phosphorus Compounds. Asymmetric Oxidation of Phosphines under Appel Conditions. J. Am. Chem. Soc. 2007, 129, 9566-9567. (e) León, T.; Riera, A.; Verdaguer, X. Stereoselective Synthesis of P-Stereogenic Aminophosphines: Ring Opening of Bulky Oxazaphospholidines. J. Am. Chem. Soc. 2011, 133, 5740-5743. (f) Berger, O.; Montchamp, J.-L. A General Strategy for the Synthesis of P-Stereogenic Compounds. Angew. Chem., Int. Ed. 2013, 52, 11377-11380. (g) Chen, T.; Han, L.-B. Optically Active H-Phosphinates and Their Stereospecific Transformations into Optically Active PStereogenic Organophosphoryl Compounds. Synlett 2015, 26, 1153-1163. (h) Rajendran, K. V.; Nikitin, K. V.; Gilheany, D. G. Hammond Postulate Mirroring Enables Enantiomeric Enrichment of Phosphorus Compounds via Two Thermodynamically Interconnected Sequential Stereoselective Processes. J. Am. Chem. Soc. 2015, 137, 9375-9381. 
(10) (a) Bauduin, C.; Moulin, D.; Kaloun, E. B.; Darcel, C.; Jugé, S. Highly Enantiomerically Enriched Chlorophosphine Boranes: Synthesis and Applications as P-Chirogenic Electrophilic Blocks. J. Org. Chem. 2003, 68, 4293-4301. (b) Bayardon, J.; Rousselin, Y.; Jugé, S. Designing P-Chirogenic 1,2-Diphosphinobenzenes at Both P-Centers Using P(III)-Phosphinites. Org. Lett. 2016, 18, 2930-2933.

(11) Imamoto, T.; Saitoh, Y.; Koide, A.; Ogura, T.; Yoshida, K. Synthesis and Enantioselectivity of P-Chiral Phosphine Ligands with Alkynyl Groups. Angew. Chem., Int. Ed. 2007, 46, 8636-8639.

(12) Gatineau, D.; Giordano, L.; Buono, G. Bulky, Optically Active P-Stereogenic Phosphine-Boranes from Pure H-Menthylphosphinates. J. Am. Chem. Soc. 2011, 133, 10728-10731.

(13) Orgué, S.; Flores-Gaspar, A.; Biosca, M.; Pàmies, O.; Diéguez, M.; Riera, A.; Verdaguer, X. Stereospecific S ${ }_{\mathrm{N}} 2 @ P$ Reactions: Novel Access to Bulky P-Stereogenic Ligands. Chem. Commun. 2015, 51, 17548-17551.

(14) Lemouzy, S.; Jean, M.; Giordano, L.; Hérault, D.; Buono, G. The Hydroxyalkyl Moiety As a Protecting Group for the Stereospecific Alkylation of Masked Secondary Phosphine-Boranes. Org. Lett. 2016, 18, 140-143.

(15) The effect of the dissociation of the P-Na bond on the configurational stability of the phosphido-borane intermediate has been highlighted in a control experiment. (See the Supporting Information, Scheme S3, page S13, for details.)

(16) The racemization could process by a radical mechanism from the iodide tert-butylphenylphosphine-borane, see: Kortmann, F. A.; Chang, M.-C.; Otten, E.; Couzijn, E. P. A.; Lutz, M.; Minnaard, A. J. Consecutive Dynamic Resolutions of Phosphine Oxides. Chem. Sci. 2014, 5, 1322-1328.

(17) Lemouzy, S.; Nguyen, D. H.; Camy, V.; Jean, M.; Gatineau, D.; Giordano, L.; Naubron, J.-V.; Vanthuyne, N.; Hérault, D.; Buono, G. Stereospecific Synthesis of $\alpha$ - and $\beta$-Hydroxyalkyl P-Stereogenic Phosphine-Boranes and Functionalized Derivatives: Evidence of the $\mathrm{P}$ $=\mathrm{O}$ Activation in the $\mathrm{BH}_{3}$-Mediated Reduction. Chem. - Eur. J. 2015, $21,15607-15621$. 